\title{
Does sacituzumab-govitecan act as a conventional antibody drug conjugate (ADC), a prodrug of SN-38 or both?
}

\author{
Daniel V. Santi ${ }^{1}$, Luc Cabel $^{2}$, François-Clément Bidard ${ }^{2,3}$ \\ ${ }^{1}$ Prolynx, San Francisco, CA, USA; ${ }^{2}$ Medical Oncology, Institut Curie, Paris, France; ${ }^{3}$ UVSQ/Paris-Saclay University, Paris, France \\ Correspondence to: François-Clément Bidard, MD, PhD. Institut Curie, 35 rue Dailly, 92210 Saint Cloud, Paris, France. Email: fcbidard@curie.fr. \\ Comment on: Bardia A, Hurvitz SA, Tolaney SM, et al. Sacituzumab Govitecan in Metastatic Triple-Negative Breast Cancer. N Engl J Med \\ 2021;384:1529-41.
}

Submitted Mar 09, 2021. Accepted for publication Jun 03, 2021.

doi: $10.21037 / \mathrm{atm}-21-1103$

View this article at: https://dx.doi.org/10.21037/atm-21-1103

On April 22, 2020, the Food and Drug Administration granted accelerated approval to sacituzumab-govitecan (Trodelvy) for adult patients with metastatic triple-negative breast cancer (TNBC) who received at least two prior therapies for metastatic disease (1). Sacituzumab-govitecan is an antibody drug conjugate (ADC) of a humanized antiTrop2 monoclonal antibody (mAb), RS7, linked to an average of 7.6 molecules of $\mathrm{SN}-38$ - the active metabolite of irinotecan and a potent inhibitor of Topoisomerase 1 (Topo1) (2). By chemically connecting the drug and $\mathrm{mAb}$ the goal was to deliver and release SN-38 to tumor cells abundant in Trop2. In the Phase III ASCENT study sacituzumab-govitecan demonstrated clinical benefit in patients with metastatic TNBC irrespective of Trop2 expression, albeit with greater efficacy in patients with a medium or high Trop2 score $(3,4)$. In contrast, although sacituzumab-govitecan was efficacious in metastatic small cell lung cancer (mSCLC), progression-free and overall survival showed no clear relationship to Trop2 expression (5). Hence, there is an enigma as to how the anti-Trop2 ADC can be effective in cancers that either have or have not the Trop2 antigen. A knowledge of the mechanism of action of sacituzumab-govitecan in Trop2 low or absent tumors would facilitate further development of SN-38-based drugs or ADCs targeting Trop2, and we present hypotheses here that explains this effect.

It was anticipated that if sacituzumab-govitecan internalized in tumor cells, a protease site on the linker would be cleaved by lysosomal enzymes to release SN-38 intracellularly. However, the internalization of sacituzumabgovitecan may not be very efficient. In early efforts to establish Trop2 targeting, tumor uptake of the carrier mAb
${ }^{131}$ I-RS7 was only $~ 7 \%$ to $16 \%$ of the initial dose/gm in a Trop2 TNBC xenograft-only $\sim 2$-fold higher than a control ${ }^{131} \mathrm{I}-\mathrm{mAb}$ (6); by comparison, Trastuzumab shows an uptake of $~ 40 \%$ of the initial dose/gm in a HER2-positive tumor (7). However, in sacituzumab-govitecan, the linker attaching the monoclonal antibody to SN-38 also contains a hydrolysable carbonate moiety that has a cleavage half-life of only $\sim 18$ hours in neutral $\mathrm{pH}$ or sera-the "weakest link in the chain". It has been suggested that the hydrolytically labile linker allows time-dependent extracellular release of free drug in the tumor microenvironment so it can affect adjacent cells by a bystander effect (2).

The rapid spontaneous linker hydrolysis in sacituzumabgovitecan releases a very large amount of the SN-38 cargo systemically (8), much more than with other ADCs-which are generally designed to avert spontaneous drug releaseor that can be accounted for by a targeted mechanism with limited target capacity. Thus, the question should be asked as to whether the antitumor effects of sacituzumabgovitecan are due to a conventional ADC mechanism, a bystander effect, systemically released $\mathrm{SN}-38$, or a combination thereof.

The Table 1 below shows the pharmacokinetic parameters of the SN-38 generated from sacituzumab-govitecan and from irinotecan-the prototypical SN-38 prodrug-in the human. Although the mechanisms of SN-38 generation are quite different, the exposure, or AUC, of the SN-38 released from sacituzumab-govitecan over a three-week cycle is over 15 -fold higher than that from irinotecan at their maximally tolerated doses; also, the time over the target (TOT) concentration of $\sim 10 \mathrm{nM} \mathrm{SN}-38$ needed to inhibit Topo1 is significantly longer with sacituzumab- 
Table 1 Pharmacokinetic parameters of SN-38 generated from sacituzumab-govitecan and irinotecan ${ }^{\mathrm{A}}$

\begin{tabular}{|c|c|c|c|c|}
\hline Drug & $\mathrm{SN}-38 \mathrm{C}_{\max }, \mathrm{nM}$ & $\mathrm{SN}-38 \mathrm{t}_{1 / 2}, \mathrm{~h}$ & 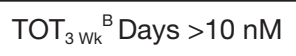 & $\mathrm{SN}-38 \mathrm{AUC}_{3 \mathrm{wk}_{k}}, \mu \mathrm{M} \times \mathrm{h}$ \\
\hline Sacituzumab-govitecan 10 mg/kg, Q Wk, 2/3 Wks & 320 & 18 & 7.5 & $20^{\mathrm{C}}$ \\
\hline Irinotecan, 340 mg/m² Q3 Wk & 140 & 21 & 4.4 & 1.2 \\
\hline
\end{tabular}

${ }^{A}$, the $C_{\max }, t_{1 / 2}$ and single-dose AUC values were obtained from the FDA label information for sacituzumab-govitecan and CPT-11 (Camptosar); ${ }^{B}$, average time SN-38 is over $10 \mathrm{nM}$ target concentration over $3 \mathrm{Wk}$; ${ }^{\mathrm{C}}$, the $\mathrm{AUC}_{3 \mathrm{wk}}$ of $\mathrm{SN}-38$ released from sacituzumabgovitecan over $3 \mathrm{Wk}$ was calculated as twice the reported $\mathrm{AUC}_{168 \mathrm{hr}}$ Wk, week; AUC, area under the curve; TOT, time on target.

govitecan. Since the much lower levels of SN-38 generated from irinotecan have significant therapeutic and toxicity effects, it can be confidently concluded that much higher systemic levels of free SN-38 released from sacituzumabgovitecan must have equal or greater pharmacological effects. This could explain the efficacy of sacituzumabgovitecan observed in low Trop2 score mSCLC (5) and mTNBC (4).

Since sacituzumab-govitecan is so effective in TNBC, one can rightly ask the importance of understanding its mechanism. First, it would certainly be important to know whether Trop2 is indeed a suitable target to either encourage or dissuade work on Trop2-targeted ADCs with different payloads. As noted above, sacituzumab-govitecan is very active in small cell lung cancer but its efficacy is unrelated to Trop2 expression (5); also, PF-06664178, a potent Trop2-targeted ADC linked to a protease-cleavable auristatin has not fared well in early clinical trials (9). Second, whether sacituzumab-govitecan is or is not a targetdirected ADC or prodrug would influence the stability of linkers chosen for similar therapeutics; DS-1062a and SKB264 are Trop2-targeting ADCs in early trials that also have Topo1 poison payloads-exatecan and belotecan, respectively-attached by protease-labile linkers that would not be released systemically and require intracellular delivery and payload release. Third, if maintenance of a high systemic concentration of free $\mathrm{SN}-38$ is essential for efficacy of sacituzumab-govitecan it may limit the use of certain drug combinations. Currently, sacituzumab-govitecan is administered IV on days 1 and 8 of a 21-day cycle, so there is a near-continuous high systemic exposure of $\mathrm{SN}-38$ with a minimal drug-free interval. PARP inhibitors are highly synergistic with Topo1 inhibitors such as $\mathrm{SN}-38$, but the synergy exists for toxicities as well as efficacy. For example, the current sacituzumab-govitecan dosing schedule does not provide a sufficient $\mathrm{SN}-38$-free interval in which a PARP inhibitor could be safely administered-as for example by using a gap schedule approach for combinations of Topo1 inhibitors and DNA damage response inhibitors (10)_ hence, the use of a sacituzumab-govitecan-PARP inhibitor combination may not be feasible. Indeed, a recent small trial of sacituzumab-govitecan and rucaparib indicated efficacy of the combination, but at the expense of significant early grade 3/4 neutropenia (11). Finally, if the high AUC of free $\mathrm{SN}-38$ is a major driver of sacituzumab-govitecan efficacy, a properly designed long-acting prodrug of SN-38 could achieve that AUC, as well as a lower $\mathrm{C}_{\max }$ to lower systemic toxicity and a prolonged half-life to increase time over target; moreover, use of a prodrug would not be confined to tumors that have Trop2. Hence, comparing the efficacy of a long-acting non-targeted SN-38 prodrug to sacituzumabgovitecan at doses that provide equal exposure may resolve to what extent sacituzumab-govitecan acts as a $\mathrm{SN}-38$ prodrug versus a targeted ADC.

\section{Conclusions}

Pharmacokinetic and biomarker data, together with considerations of its rapidly hydrolyzed linker, suggests that sacituzumab-govitecan might act as an $\mathrm{SN}$-38-prodrug instead of or in addition to a conventional ADC.

\section{Acknowledgments}

Funding: None.

\section{Footnote}

Provenance and Peer Review: This article was a standard submission to the journal. The article has undergone external peer review.

Peer Review File: Available at https://dx.doi.org/10.21037/ atm-21-1103

Conflicts of Interest: All authors have completed the ICMJE uniform disclosure form (available at https://dx.doi. org/10.21037/atm-21-1103). DVS is the co-founder and 
President of Prolynx, which developed PLX038. LC and FCB will act as investigators in a forthcoming clinical trial of PLX038. The authors have no other conflicts of interest to declare.

Ethical Statement: The authors are accountable for all aspects of the work in ensuring that questions related to the accuracy or integrity of any part of the work are appropriately investigated and resolved.

Open Access Statement: This is an Open Access article distributed in accordance with the Creative Commons Attribution-NonCommercial-NoDerivs 4.0 International License (CC BY-NC-ND 4.0), which permits the noncommercial replication and distribution of the article with the strict proviso that no changes or edits are made and the original work is properly cited (including links to both the formal publication through the relevant DOI and the license). See: https://creativecommons.org/licenses/by-nc-nd/4.0/.

\section{References}

1. Bardia A, Mayer IA, Vahdat LT, et al. Sacituzumab Govitecan-hziy in Refractory Metastatic Triple-Negative Breast Cancer. N Engl J Med 2019;380:741-51.

2. Goldenberg DM, Sharkey RM. Sacituzumab govitecan, a novel, third-generation, antibody-drug conjugate (ADC) for cancer therapy. Expert Opin Biol Ther 2020;20:871-85.

3. Hurvitz SA, Tolaney SM, Punie K, et al. Abstract GS306: Biomarker evaluation in the phase 3 ASCENT study of sacituzumab govitecan versus chemotherapy in patients with metastatic triple-negative breast cancer. Cancer Res

Cite this article as: Santi DV, Cabel L, Bidard FC. Does sacituzumab-govitecan act as a conventional antibody drug conjugate (ADC), a prodrug of SN-38 or both? Ann Transl Med 2021;9(14):1113. doi: 10.21037/atm-21-1103
2021;81:GS3-06.

4. Bardia A, Hurvitz SA, Tolaney SM, et al. Sacituzumab Govitecan in Metastatic Triple-Negative Breast Cancer. N Engl J Med 2021;384:1529-41.

5. Gray JE, Heist RS, Starodub AN, et al. Therapy of Small Cell Lung Cancer (SCLC) with a Topoisomerase-Iinhibiting Antibody-Drug Conjugate (ADC) Targeting Trop-2, Sacituzumab Govitecan. Clin Cancer Res 2017;23:5711-9.

6. Shih LB, Xuan H, Aninipot R, et al. In vitro and in vivo reactivity of an internalizing antibody, RS7, with human breast cancer. Cancer Res 1995;55:5857s-63s.

7. Lewis Phillips GD, Nishimura MC, Lacap JA, et al. Trastuzumab uptake and its relation to efficacy in an animal model of HER2-positive breast cancer brain metastasis. Breast Cancer Res Treat 2017;164:581-91.

8. Ocean AJ, Starodub AN, Bardia A, et al. Sacituzumab govitecan (IMMU-132), an anti-Trop-2-SN-38 antibody-drug conjugate for the treatment of diverse epithelial cancers: Safety and pharmacokinetics. Cancer 2017;123:3843-54.

9. King GT, Eaton KD, Beagle BR, et al. A phase 1, doseescalation study of PF-06664178, an anti-Trop-2/ Aur0101 antibody-drug conjugate in patients with advanced or metastatic solid tumors. Invest New Drugs 2018;36:836-47.

10. Thomas A, Pommier Y. Targeting Topoisomerase I in the Era of Precision Medicine. Clin Cancer Res 2019;25:6581-9.

11. Yap TA, Hamilton EP, Bauer TM, et al. 547P Rucaparib + sacituzumab govitecan (SG): Initial data from the phase Ib/II SEASTAR study (NCT03992131). Ann Oncol 2020;31:S462-504. 